\title{
A Giant, Complex Fibroepithelial Tumor of the Breast: Borderline Phyllodes Tumor Combined with Tubular Adenoma - a Rare Clinical Presentation of a Fibroepithelial Tumor of the Breast
}

\author{
Nermina Ibisevic ${ }^{1}$, Jasmina Bajrovic ${ }^{2}$, Ena Saranovic ${ }^{1}$, Dijana Spiritovic ${ }^{1}$, Faruk Skenderi ${ }^{1}$, Semir Vranic ${ }^{3,4, a}$ \\ ${ }^{1}$ Department of Pathology, University Clinical Center Sarajevo, Sarajevo, Bosnia and Herzegovina, ${ }^{2}$ Department of Radiology, \\ University Clinical Center Sarajevo, Sarajevo, Bosnia and Herzegovina, ${ }^{3}$ College of Medicine, QU Health, Qatar University, \\ Doha, Qatar, ${ }^{4}$ Biomedical and Pharmaceutical Research Unit, QU Health, Qatar University, Doha, Qatar
}

Correspondence: semir.vranic@gmail.com or svranic@qu.edu.qa; Tel.: + 9744403 7873; Fax.: + 97444032250

Received: 5 February 2021; Accepted: 12 February 2021

Key Words: Breast • Fibroepithelial Tumors • Phyllodes Tumor • Tubular Adenoma.

A 65-year-old woman with a negative family history of breast cancer presented with a palpable mass in the left breast's central portion. Mammography revealed an oval heteroechogenic, partly solid, partly cystic, sharply demarcated mass, measuring $100 \times 90 \mathrm{~mm}$ in greatest diameter, classified as BI-RADS 4c, according to ACR BI-RADS Atlas Fifth Edition (Figure 1A-B). Breast MRI showed a lobulated mass with smooth margins appearing hypointense on T1WI and high signal intensity on T2WI (Figure 1C-D). A core needle biopsy revealed a cellular neoplasm, composed of small, closely packed tubules with spindle cell intervening stroma without prominent atypia and mitotic activity, classified as B3 category according to the UK National Coordinating Committee for Breast Screening Pathology (Figure 2A). The multidisciplinary tumor board discussed the case and recommended a wide surgical excision. With the patient's approval, a left mastectomy was recommended and performed. The axillary clearance was not performed. The $100 \times 90 \mathrm{~mm}$ tumor was grossly well-circumscribed, grayish-white, and predominantly solid, with a smaller cystic component, without necrosis and hemorrhage (Figure $2 \mathrm{~B}$ ). Histopathologic examination revealed a well-circumscribed tumor with two distinct components (tubular adenoma and phyllodes tumor) with the transition to one another (Figure 2C). The larger portion of the tumor was composed of closely packed small round to oval tubules with little intervening spindle cell stroma consistent with tubular adenoma. The smaller component showed a biphasic fibroepithelial tumor with leaf-like projections with moderately cellular stroma (Figure 2D-E). The stromal cells exhibited mild to moderate atypia, and their mitotic activity was up to six mitoses/10 hpf (Figure 2F). Stromal overgrowth was absent, while the malignant heterologous elements were not observed despite the exhaustive tumor sampling (25 paraffin blocks). The final diagnosis was a complex fibroepithelial tumor composed of borderline phyllodes and tubular adenoma.

aORCID: http://orcid.org/0000-0001-9743-7265 

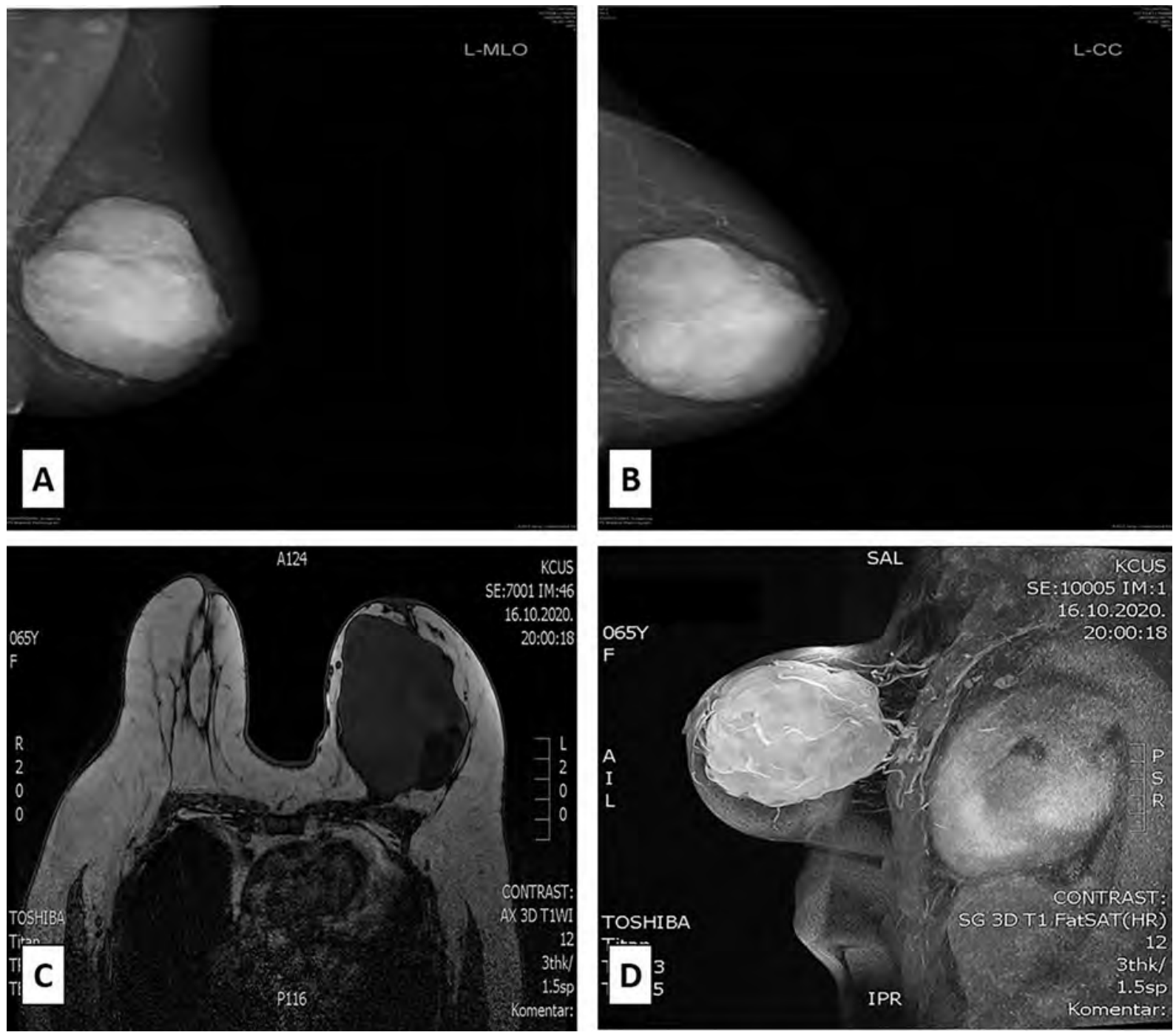

Figure 1A-D. Mammograms on the standard view (MLO and CC) revealed a round, dense mass partially surrounded by a clear halo, without any calcifications (A-B); Axial T1W1 MRI scans revealed a hypointense mass with some hyperintense foci of hemorrhage (C); Sagittal post-gadolinium subtraction image also showed intense enhancement (D). 

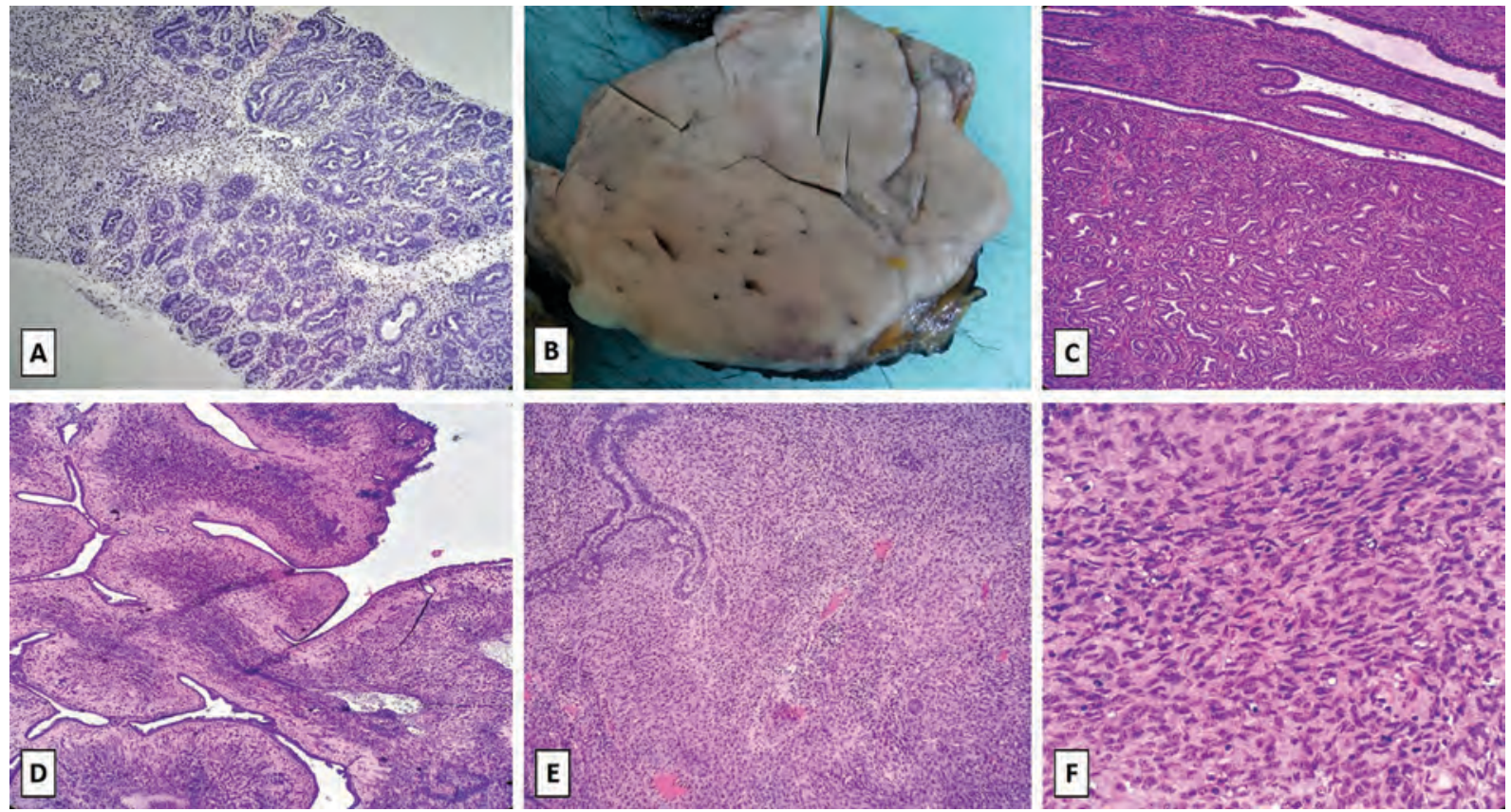

Figure 2A-F. A core biopsy specimen revealed predominantly tubular adenoma component with some spindle cell intervening stroma (Hematoxylin and Eosin, 10X) (A); A gross specimen revealed a large, $(10 \times 9 \mathrm{~cm})$, well-demarcated solid mass without hemorrhage and necrosis (B); Microscopic examination revealed two distinct fibroepithelial tumors: tubular adenoma and phyllodes tumor with the abrupt transition one to another (Hematoxylin and Eosin, 10x) (C); Leaf-like projections, a hallmark of phyllodes tumor of the breast, were easily identified in the phyllodes part of the mass (Hematoxylin and Eosin, 10x) (D); The stromal component exhibited moderate cellularity without stromal overgrowth (Hematoxylin and Eosin, 10x) (E); The stromal cells showed moderate atypia with up to six mitotic figures/10 hpf (Hematoxylin and Eosin, 20x) (F).

\section{Ethical Approval}

The local institutional review board has the policy not to review the case studies. All the procedures reported in the current manuscript were performed according to the Declaration of Helsinki.

Conflict of Interest: The authors declare that they have no conflict of interest. 